\title{
Expression of human epidermal growth factor receptor 2 in primary and paired parenchymal recurrent and/or metastatic sites of gastric cancer
}

\author{
RYOSUKE SHIBATA $^{1}$, SATOSHI NIMURA ${ }^{2}$, TATSUYA HASHIMOTO ${ }^{1}$, TORU MIYAKE ${ }^{1}$, \\ SHINSUKE TAKENO $^{1}$, SEIICHIRO HOSHINO ${ }^{1}$, KAZUKI NABESHIMA ${ }^{2}$ and YUICHI YAMASHITA ${ }^{1}$ \\ Departments of ${ }^{1}$ Gastroenterological Surgery and ${ }^{2}$ Pathology, \\ Fukuoka University Faculty of Medicine, Fukuoka 814-0180, Japan
}

Received May 13, 2014; Accepted June 10, 2014

DOI: $10.3892 / \mathrm{mco} .2014 .329$

\begin{abstract}
Human epidermal growth factor receptor 2 (HER2) status has been evaluated at the primary site of gastric cancer when planning trastuzumab therapy against recurrent or metastatic lesions, since tissue sampling is uncommon in recurrent or metastatic lesions. This study retrospectively investigated the concordance of HER2 expression between primary and metastatic/recurrent lesions in order to confirm sensitivity to trastuzumab. The subjects comprised 37 patients with gastric adenocarcinoma who underwent tissue biopsy or surgical resection of the primary sites and 49 paired synchronous or metachronous metastatic sites (excluding lymph nodes) at the Fukuoka University Hospital between January, 1998 and September, 2012. All the samples were evaluated for HER2 status at the invasive front by immunohistochemistry (IHC). The HER 2 positivity rate of the primary sites was $\sim 16 \%$ and the concordance ratio of the IHC results between primary and paired metastatic sites was $\sim 97 \%$. No discordant cases regarding HER2 status were found among metachronous interventions for metastatic lesions. Only one patient exhibited conversion from a HER2-negative status in all the portions of the primary site to a positive status in a metastatic site. In conclusion, a high concordance ratio for HER2 status was observed between primary and paired metastatic lesions. Thus, employing trastuzumab therapy against metastatic or recurrent gastric cancer based on the HER2 status of the primary lesion appears to be an acceptable approach.
\end{abstract}

Correspondence to: Dr Ryosuke Shibata, Department of Gastroenterological Surgery,Fukuoka University Faculty of Medicine, Nanakuma 7-45-1, Jonan-ku, Fukuoka 814-0180, Japan

E-mail: ryosukeshibata@hotmail.com

Key words: human epidermal growth factor receptor 2, concordance, multiple intervention, inoperable gastric cancer

\section{Introduction}

Gastric cancer is one of the most commonly diagnosed cancers and the second most common cause of cancer-related mortality worldwide (1,2). Radical gastrectomy and lymph node dissection with adjuvant chemotherapy are performed for patients with advanced gastric cancer (3). However, metastatic gastric cancer has a 5-year survival rate of 5-20\% and a median overall survival of $<1$ year $(1,4,5)$. In 2010 , trastuzumab combined with chemotherapy was established as a new standard treatment option for human epidermal growth factor receptor 2 (HER2)-positive advanced gastric or gastroesophageal junction cancer by the ToGA study (6). Although trastuzumab combination therapy is adopted for inoperable advanced or metastatic disease, the HER 2 status is commonly evaluated in the primary lesion, since metastatic sites are rarely resected or biopsied prior to treatment. With breast cancer, however, the concordance ratio for HER2 status between the primary lesion and metastatic lymph nodes was reported to be $90-98 \%(7,8)$, whereas the concordance ratio for HER2 status between primary and metastatic sites other than lymph nodes was reported to be lower $(9,10)$. However, although a high concordance ratio for HER2 status between primary and lymph node lesions has been reported in gastric cancer $(11,12)$, a concordance ratio for HER2 status between primary and metastatic lesions other than lymph nodes has not been reported. In addition, HER2 status is typically evaluated by immunohistochemistry (IHC) and/or fluorescence in situ hybridization (FISH) and a high concordance ratio between IHC and FISH has been reported (13).

In this study, HER 2 expression was assessed using IHC (IHC score 2+) and FISH in the primary lesion and in paired metastatic lesions other than lymph nodes. The aim of this study was to investigate the concordance of HER2 expression between primary and metastatic lesions and the feasibility of using HER 2 expression in the primary lesion for determining therapy against metastatic lesions.

\section{Patients and methods}

Patients and tissue samples. The samples used in this study were surgically resected or biopsied at Fukuoka University 
Table I. Clinicopathological characteristics of the study population.

\begin{tabular}{|c|c|c|c|}
\hline Characteristics & $\begin{array}{c}\text { Total } \\
(\%)\end{array}$ & $\begin{array}{c}\text { No. of } \\
\text { primary } \\
\text { sites }(\%) \\
(\mathrm{n}=37)\end{array}$ & $\begin{array}{c}\text { No. of } \\
\text { metastatic } \\
\text { sites }(\%) \\
(\mathrm{n}=49)\end{array}$ \\
\hline \multicolumn{4}{|l|}{ Age (years) } \\
\hline Median & 64 & & \\
\hline Range & $45-80$ & & \\
\hline \multicolumn{4}{|l|}{ Gender } \\
\hline Male & $30(81.0)$ & & \\
\hline Female & $7(19.0)$ & & \\
\hline \multicolumn{4}{|l|}{ Type of intervention } \\
\hline Surgical resection & $75(87.0)$ & $28(76.0)$ & $47(96.0)$ \\
\hline Biopsy & $11(13.0)$ & $9(24.0)$ & $2(4.0)$ \\
\hline \multicolumn{4}{|l|}{ Histological type } \\
\hline Differentiated & $13(35.0)$ & & \\
\hline Undifferentiated & $17(46.0)$ & & \\
\hline Mixed & $7(19.0)$ & & \\
\hline \multicolumn{4}{|l|}{ Metastasis } \\
\hline Synchronous & $19(39.0)$ & & \\
\hline Metachronous & $30(61.0)$ & & \\
\hline \multicolumn{4}{|l|}{$\begin{array}{l}\text { Pre-intervention } \\
\text { chemotherapy of } \\
\text { metastatic sites }\end{array}$} \\
\hline UFT & $9(18.0)$ & & \\
\hline S-1 & $9(18.0)$ & & \\
\hline Others & $5(10.0)$ & & \\
\hline None & $24(54.0)$ & & \\
\hline
\end{tabular}

UFT, uracil-tegafur; S-1, tegafur-gimeracil-oteracil potassium.

Hospital between January, 1998 and September, 2012. A total of 37 patients with gastric adenocarcinoma (9 biopsies and 28 resection specimens) and 49 paired synchronous or metachronous metastatic tissues ( 2 biopsies and 47 resection specimens) were analyzed. The invasive front of the resected tumor tissues was examined immunohistochemically. None of the patients received neoadjuvant therapy and 23 metastatic tissue samples were obtained from patients who had been treated with chemotherapy. No patients received trastuzumab combination therapy. Metachronous metastasis was defined as metastasis arising $>6$ months following curative resection.

HER2 expression and amplification. HER2 status was examined using $10 \%$ formalin-fixed paraffin-embedded tissues. Immunohistochemical staining was performed automatically with the Ventana iView PATHWAY HER2 (4B5) (Ventana Medical Systems, Roche, Tucson, AZ, USA). Antigen activation was performed in citrate buffer under high pressure. HER2 immunoreactivity was scored as negative ( 0 or $1+)$, equivocal $(2+)$ and positive $(3+)$ by an experienced pathologist according to the scoring system described by Hofmann et al (14).
Table II. Human epidermal growth factor receptor 2 (HER2) status of primary and metastatic sites.

\begin{tabular}{|c|c|c|c|c|c|}
\hline \multirow[b]{3}{*}{ HER2 status } & \multicolumn{5}{|c|}{ Metastatic sites } \\
\hline & \multicolumn{3}{|c|}{ Negative } & \multicolumn{2}{|c|}{ Positive } \\
\hline & 0 & $1+$ & $2+(\mathrm{FISH}-)$ & $2+(\mathrm{FISH}+)$ & $3+$ \\
\hline \multicolumn{6}{|l|}{ Primary sites } \\
\hline \multicolumn{6}{|l|}{ Negative } \\
\hline 0 & 28 & 1 & 0 & 0 & 1 \\
\hline $1+$ & 1 & 0 & 0 & 0 & 0 \\
\hline $2+($ FISH-) & 0 & 0 & 0 & 0 & 0 \\
\hline \multicolumn{6}{|l|}{ Positive } \\
\hline $2+(\mathrm{FISH}+)$ & 0 & 0 & 0 & 0 & 1 \\
\hline $3+$ & 0 & 0 & 0 & 0 & 5 \\
\hline
\end{tabular}

FISH, fluorescence in situ hybridization.

Table III. Association of human epidermal growth factor receptor 2 (HER2) status of the primary site with histological type and type of intervention.

\begin{tabular}{lccc}
\hline & \multicolumn{2}{c}{ HER2 status } & \\
\cline { 2 - 3 } Variables & Negative & Positive & P-value \\
\hline Histological type & & & 0.0244 \\
Differentiated & 8 & 5 & \\
Undifferentiated & 16 & 1 & \\
Mixed & 7 & 0 & \\
Type of intervention & & & $>0.9999$ \\
Surgical resection & 23 & 5 & \\
Biopsy & 8 & 1 & \\
\hline
\end{tabular}

Table IV. Concordance of human epidermal growth factor receptor 2 (HER2) status between primary and paired metastatic lesions.

\begin{tabular}{lccc}
\hline & \multicolumn{2}{c}{ Metastatic site } & \\
\cline { 2 - 3 } HER2 status & Negative & Positive & P-value \\
\hline $\begin{array}{l}\text { Primary site } \\
\text { Negative }\end{array}$ & 30 & 1 & $<0.0001$ \\
Positive & 0 & 6 & \\
\hline
\end{tabular}

The PathVysion HER2 DNA Probe kit (Abbott Molecular, Abbott Park, IL, USA) and a BioView Duet-3 scanning system (BioView, Ltd., Rehovot, Israel) with fluorescence microscopy (BX51 TRF; Olympus, Nagano, Japan) were used for FISH. Gene amplification was scored when a minimum of 20 cancer cell nuclei exhibited a HER2/chromosome enumeration probe (CEP)17 ratio of $>2$. 


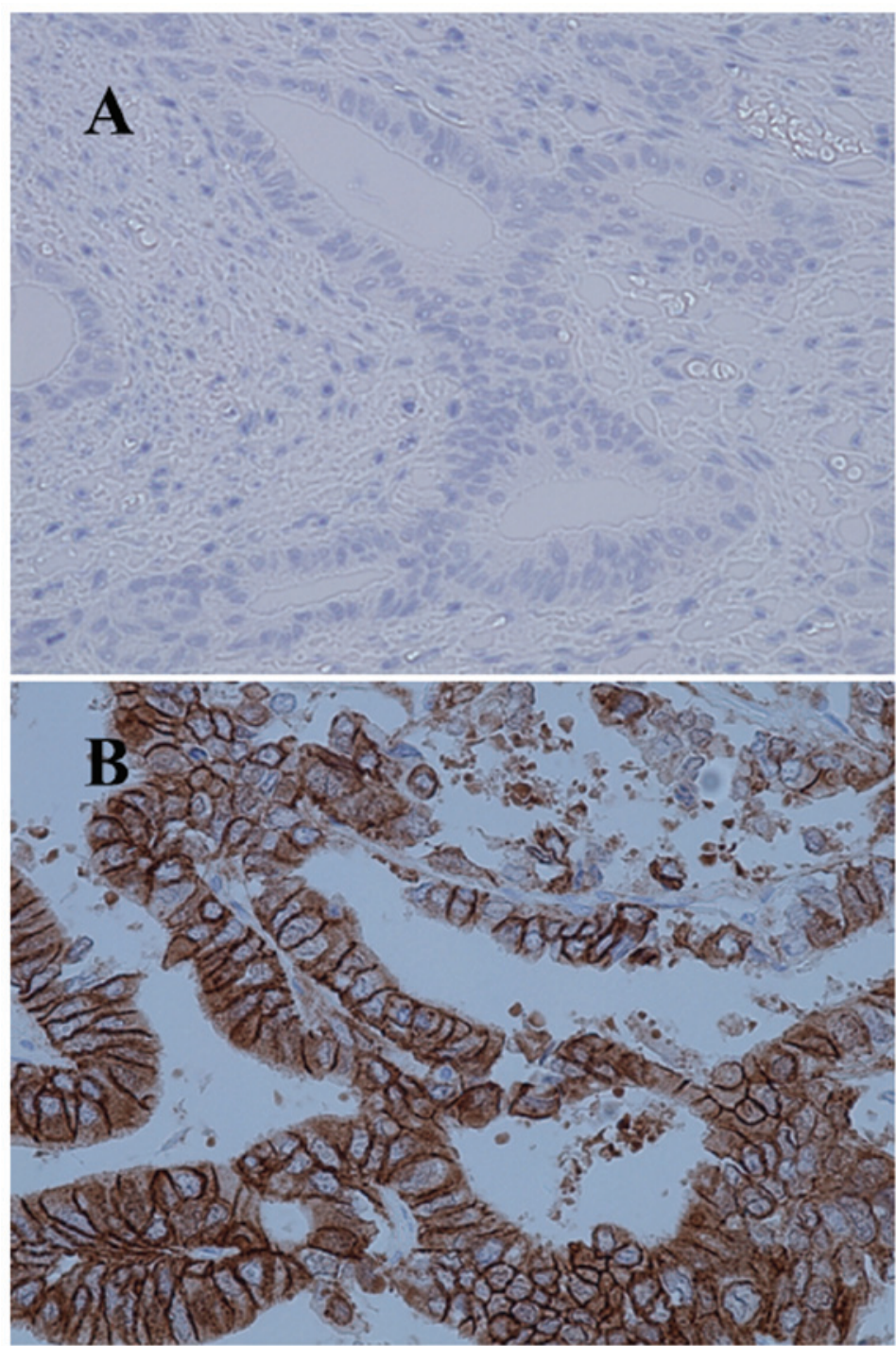

Figure 1. Human epidermal growth factor receptor 2 (HER2) expression by immunohistochemistry (A) at the primary site and (B) at paired metastatic sites. The histological type at the primary site was moderately differentiated tubular adenocarcinoma and HER 2 status was scored as 0 . The metastatic site was the liver and HER2 status was scored as 3+. Magnification, $\mathrm{x} 40$.

HER2-positive status was defined as IHC $3+$, or HER2 2+ and FISH-positive (HER2/CEP17 ratio $>2$ ). HER2-negative status was defined as IHC $0,1+$, or $2+$ and FISH-negative; the positive groups were considered suitable for trastuzumab combination therapy (6). All IHC 2+ tumors were further analyzed with FISH to determine the HER2 gene copy level. All the tissue samples from metastatic sites enabled the pathologist to confirm the lesions as being metastatic from gastric cancer.

Statistical methods. For the evaluation of the correlations of HER2 status between primary and paired metastatic lesion, the Fisher's exact probability test was employed. $\mathrm{P}<0.05$ was considered to indicate a statistically significant difference.

\section{Results}

Clinicopathological characteristics. The clinicopathological characteristics of the patients in this study are summarized in Table I. The metastatic sites included the peritoneum $(n=20)$, liver $(n=10)$, lung $(n=7)$, skin and subcutaneous tissue $(n=5)$, colon $(n=3)$ and others $(n=4)$ (data not shown).

HER2 IHC. The results for HER2 IHC are shown in Table II. The HER 2 status of the primary sites was scored as 0 in 30 specimens $(81 \%), 1+$ in $1(3 \%), 2+$ in $1(3 \%)$ and $3+$ in $5(13 \%)$, while metastatic sites were scored as 0 in 29 specimens (78\%), $1+$ in $1(3 \%), 2+$ in $0(0 \%)$ and $3+$ in $7(19 \%)$. The HER 2 positivity (IHC $3+$ or $2+$ and FISH-positive) ratio of primary sites was $\sim 16 \%$. The association of the HER2 status of the primary site with histological type and type of intervention are shown in Table III. The HER2 positivity ratio of the differentiated type was significantly higher compared to that of the undifferentiated type. However, there was no significant association between the HER2 status of the primary site and the type of intervention. The total concordance ratio between primary sites and metastatic sites was $\sim 97 \%$ (Table IV), reflecting a significant correlation $(\mathrm{P}<0.0001)$. Only one case exhibited positive conversion (Fig. 1A and B). This case evaluated HER 2 status between primary and paired metastatic sites 
Table V. Human epidermal growth factor receptor 2 (HER2) status with metachronous interventions.

\begin{tabular}{|c|c|c|c|c|c|c|c|}
\hline \multirow[b]{2}{*}{ Case } & \multirow[b]{2}{*}{ Histology } & \multirow{2}{*}{$\begin{array}{l}\text { HER2 status } \\
\text { of the } \\
\text { primary site }\end{array}$} & \multicolumn{5}{|c|}{ HER2 status in metachronous interventions } \\
\hline & & & 1 st & 2nd & $3 \mathrm{rd}$ & 4th & 5 th \\
\hline 1 & Differentiated & 0 & 0: Colon & 0: Skin & 0 : Skin & 0: Kidney & 0 : Skin \\
\hline 2 & Undifferentiated & 0 & 0: Colon & 0: Peritoneum & & & \\
\hline 3 & Undifferentiated & 0 & 0: Colon & 0: Liver & & & \\
\hline 4 & Differentiated & 0 & 3+: Liver & 3+: Peritoneum & & & \\
\hline 5 & Undifferentiated & 0 & 0: Liver & 0: Peritoneum & & & \\
\hline 6 & Differentiated & $3+$ & 3+: Lung & 3+: Lung & & & \\
\hline 7 & Differentiated & $3+$ & 3+: Cerebellum & 3+: Lung & & & \\
\hline 8 & Undifferentiated & 0 & 0: Ovary & 0 : Skin & & & \\
\hline 9 & Undifferentiated & 0 & 0: Peritoneum & 0: Peritoneum & & & \\
\hline
\end{tabular}

by resected specimen. Although in this case all the portions of the primary site were examined, no positive reaction was observed on the membrane in any of the specimens. The equivocal (2+) case exhibited amplification by FISH and was therefore classified as positive. Although one case of discordance between primary and metastatic sites was identified, there were no discordances among metachronous metastatic sites (Table V).

\section{Discussion}

Previous studies have estimated the HER 2 positivity ratio to be $8 \cdot 1-17.1 \%$ in gastric cancer $(6,15,16)$. Recent studies reported that the HER2 positivity ratio is lower in patients with curatively resectable gastric cancer compared to that in unresectable patients $(15,16)$. The present study estimated an HER2-positive ratio of $\sim 16 \%$, presumably because the study population comprised patients with curatively resected and unresected or recurrent gastric cancer. In this study, the differentiated type of tumor exhibited a significantly high HER2 positivity, as the consensus reported that the majority of positive cases were histologically of the intestinal type (17). However, Lee et al (18) reported a discordance of the HER2 IHC score between biopsies and gastrectomies; in this study, there was no difference between bioptic and resected specimens, although only a small series was analyzed.

The HER2 status in gastric cancer is commonly evaluated immunohistochemically, with a IHC $2+$ status further analyzed by FISH, since previous studies demonstrated a high concordance ratio between IHC and FISH in gastric cancer $(11,13,14,17,19,20)$ and the American Society of Clinical Oncology/College of American Pathologists guidelines recommend that FISH analysis be conducted for cases with an IHC 2+ lesion in breast cancer (21). In gastric cancer, combination chemotherapy with trastuzumab was adopted from 2010 onwards and sufficient scientific evidence regarding HER2 has not yet been accumulated. Therefore, references have been made to previous studies regarding HER2 in breast cancer. Since metastatic sites from gastric cancer patients are rarely resected or biopsied, evidence on the concordance of HER2 status by IHC between primary tumor and paired metastatic lesions other than lymph nodes has not generally been reported $(17,22)$. In breast cancer, a high concordance ratio between primary and matched metastatic lymph nodes has been reported $(7,8)$. However, in parenchymal metastases, the concordance ratio was found to be lower $(9,10)$. Nakamura et al (23) reported that biopsy of the metastatic lesions may be useful for determining treatment strategies. We examined the tumor invasive front at the primary sites in resected specimens, as it was previously reported that HER2 staining exhibits no preferential distribution within the tumor, with negligible variation between the tumor mucosal surface and the invasive front (24) and the tumor invasive front is closely involved in the metastatic process. However, Fusco et al (25) reported that there is discordance in the HER2 status between the tumor invasive front and other lesions and gastric cancer is known to exhibit heterogeneous HER2 expression $(14,18)$. Therefore we examined all the sites of the primary tumor in the discordant case to determine whether there was heterogeneity of HER2 expression, in order to assess the discordance between primary and paired metastatic lesions. However, all the lesions were IHC-scored as 0. In addition, we investigated whether there exists discordance among metachronous multiple interventions. A limited number of studies $(17,22,26)$ have addressed such issues in gastric cancer. Kim et al (17) reported significant discordance (13.1\%) between primary and metastatic lesions by IHC, but no discordance by FISH. Bozzetti et al (22) reported a concordance ratio of $94.9 \%$ between primary and matched metastatic lesions by IHC. In addition, Kochi et al (26) reported a discordance ratio of $9.8 \%$ in the HER2 status between primary sites and metastatic lymph nodes by FISH and IHC. Our results using IHC revealed a high concordance ratio $(\sim 97 \%)$ between primary and paired metastatic lesions. These results suggest the efficacy of HER2 status examination in the primary lesion for assessing the status of parenchymal metastatic lesions. Only one case of positive conversion was found in our study; likewise, Bozzetti et al (22) reported only a single case of discordance between the primary lesion and metastasis. The discordant case in this study was IHC 0 at the 
primary site and underwent liver resection for metachronous liver recurrence following hepatic intra-arterial chemotherapy (fluorouracil + cisplatin + irinotecan). The HER2 status of the liver specimen at that time was IHC 3+ and the HER2 status of the metachronous peritoneal recurrent specimen was also IHC $3+$. These findings suggest that the transition of the metastatic process strongly involves the discordance of HER2 status between primary and metastatic sites rather than heterogeneity of HER2 expression within the primary lesion. Further investigation of this issue in a larger series is required.

Nine patients underwent metachronous multiple interventions for metastatic lesions; no cases of discordance during the therapeutic period were encountered. To the best of our knowledge, no previous studies have reported such findings.

Unfortunately, no cases in this study population received trastuzumab combination therapy and further investigation, including those cases, is required.

In conclusion, the concordance ratio for HER2 status between primary and parenchymal metastatic or recurrent lesions was high. Therefore, determining the HER2 status in the primary lesion may be acceptable when considering the suitability of anti-HER 2 agents for patients with inoperable advanced or recurrent gastric cancer.

\section{Acknowledgements}

The authors wish to thank Yuki Mutsumi for assisting with IHC and FISH.

\section{References}

1. Kamangar F, Dores GM and Anderson WF: Patterns of cancer incidence, mortality, and prevalence across five continents: defining priorities to reduce cancer disparities in different geographic regions of the world. J Clin Oncol 24: 2137-2150, 2006.

2. Parkin DM, Bray F, Ferlay J and Pisani P: Global cancer statistics, 2002. CA Cancer J Clin 55: 74-108, 2005.

3. Otsuji E, Yamaguchi T, Sawai K, et al: Recent advances in surgical treatment have improved the survival of patients with gastric carcinoma. Cancer 82: 1233-1237, 1998.

4. Horner MJ, Ries LAG, Krapcho M, et al: SEER Cancer Statistics Review, 1975-2006. http://seer.cancer.gov/archive/ csr/1975_2006/results_merged/sect_24_stomach.pdf. Accessed April 12, 2009

5. Cunningham SC, Kamangar F, Kim MP, et al: Survival after gastric adenocarcinoma serection: eighteen-year experience at a single institution. J Gastrointest Surg 9: 718-725, 2005.

6. Bang YJ, Van Cutsem E, Feyereislova A, et al: Trastuzumab in combination with chemotherapy versus chemotherapy alone for treatment of HER2-positive advanced gastric or gastro-oesophageal junction cancer (ToGA): a phase 3 , open-label, randomized controlled trial. Lancet 376: 687-697, 2010.

7. Cardoso F, Di Leo A, Larsimont D, et al: Evaluation of HER2, p53, bcl-2, topoisomerase II-alpha, heat shock proteins 27 and 70 in primary breast cancer and metastatic ipsilateral axillary lymph nodes. Ann Oncol 12: 615-620, 2001.

8. Simon R, Nocito A, Huebscher T, et al: Patterns of her-2/neu amplification and overexpression in primary and metastatic breast cancer. J Natl Cancer Inst 93: 1141-1146, 2001.
9. Chang HJ, Han SW, Oh DY, et al: Discordant human epidermal growth factor receptor 2 and hormone receptor status in primary and metastatic breast cancer and response to trastuzumab. Jpn J Clin Oncol 41: 593-599, 2011.

10. Chan A, Morey A, Brown B, et al: A retrospective study investigating the rate of HER2 discordance between primary breast carcinoma and locoregional or metastatic disease. BMC Cancer 12: 555, 2012.

11. Marx AH, Tharun L, Muth J, et al: HER-2 amplification is highly homogenous in gastric cancer. Hum Pathol 40: 769-777, 2009.

12. Fassan M, Ludwig K, Pizzi M, et al: Human epithelial growth factor receptor 2 (HER2) status in primary and metastatic esophagogastric junction adenocarcinomas. Hum Pathol 43: 1206-1212, 2012

13. Tafe LJ, Janjigian YY, Zaidinski M, et al: Human epidermal growth factor receptor 2 testing in gastroesophageal cancer: correlation between immunohistochemistry and fluorescence in situ hybridization. Arch Pathol Lab Med 135: 1460-1465, 2011.

14. Hofmann M, Stoss O, Shi D, et al: Assessment of a HER2 scoring system for gastric cancer: results from a validation study. Histopathology 52: 797-805, 2008.

15. Kunz PL, Mojtahed A, Fisher GA, et al: HER2 expression in gastric and gastroesophageal junction adenocarcinoma in a US population: clinicopathologic analysis with proposed approach to HER2 assessment. Appl Immunohistochem Mol Morphol 20: 13-24, 2012.

16. Kataoka Y, Okabe H, Yoshizawa A, et al: HER2 expression and its clinicopathological features in resectable gastric cancer. Gastric Cancer 16: 84-93, 2013

17. Kim MA, Lee HJ, Yang HK, et al: Heterogeneous amplification of ERBB2 in primary lesions is responsible for the discordant ERBB2 status of primary and metastatic lesions in gastric carcinoma. Histopathology 59: 822-831, 2011.

18. Lee S, de Boer WB, Fermoyle S, et al: Human epidermal growth factor 2 testing in gastric carcinoma: issues related to heterogeneity in biopsies and resections. Histopathology 59: 832-840, 2011.

19. Takehara T, Kunitomo K, Kono K, et al: Status of c-erbB-2 in gastric adenocarcinoma: a comparative study of immunohistochemistry, fluorescence in situ hybridization and enzyme-linked immuno-sorbent assay. Int J Cancer 98: 833-837, 2002.

20. Yano T, Doi T, Ohtsu A, et al: Comparison of HER2 gene amplification assessed by fluorescence in situ hybridization and HER2 protein expression assessed by immunohistochemistry in gastric cancer. Oncol Rep 15: 65-71, 2006.

21. Wolff AC, Hammond ME, Schwartz JN, et al; American Society of Clinical Oncology; College of American Pathologists: American Society of Clinical Oncology/College of American Pathologists guideline recommendations for human epidermal growth factor receptor 2 testing in breast cancer. J Clin Oncol 25: 118-145, 2007.

22. Bozzetti C, Negri FV, Lagrasta CA, et al: Comparison of HER2 status in primary and paired metastatic sites of gastric carcinoma. Br J Cancer 104: 1372-1376, 2011.

23. Nakamura R, Yamamoto N, Onai Y, et al: Importance of confirming HER2 overexpression of recurrence lesion in breast cancer patients. Breast Cancer 20: 336-341, 2013.

24. Kim KC, Koh YW, Chang HM, et al: Evaluation of HER2 protein expression in gastric carcinomas: comparative analysis of 1,414 cases of whole-tissue sections and 595 cases of tissue microarrays. Ann Surg Oncol 18: 2833-2840, 2011.

25. Fusco N, Rocco EG, Del Conte C, et al: HER2 in gastric cancer: a digital image analysis in pre-neoplastic, primary and metastatic lesions. Mod Pathol 26: 816-824, 2013.

26. Kochi M, Fujii M, Masuda S, et al: Differing deregulation of HER2 in primary gastric cancer and synchronous related metastatic lymph nodes. Diagn Pathol 8: 191, 2013. 